\title{
ONE-PARAMETER INVERSE SEMIGROUPS
}

\author{
BY \\ CARL EBERHART AND JOHN SELDEN
}

\begin{abstract}
This is the second in a projected series of three papers, the aim of which is the complete description of the closure of any one-parameter inverse semigroup in a locally compact topological inverse semigroup. In it we characterize all one-parameter inverse semigroups. In order to accomplish this, we construct the free one-parameter inverse semigroups and then describe their congruences.
\end{abstract}

0 . Let $G$ be a subgroup of the multiplicative group of positive real numbers and let $P$ denote the subsemigroup of $G$ consisting of all $x \in G$ with $x \geqq 1$. Denote by $\mathscr{C}_{P}$ the class of all inverse semigroups $H$ for which there is a homomorphism $f: P \rightarrow H$ such that $f(P)$ generates $H$ (no proper inverse subsemigroup of $H$ contains $f(P)$ ). We shall call such semigroups $H$ one-parameter inverse semigroups and denote by $\mathscr{C}=\bigcup_{P} \mathscr{C}_{P}$ the class of all one-parameter inverse semigroups.

The class $\mathscr{C}$ contains well-known semigroups. For example, each homomorphic image of a subgroup of $R$, the positive real numbers, is a member of $\mathscr{C}$. Also the bicyclic semigroup $B$ is a member of $\mathscr{C}$, as is seen by noting that $B$ is generated by a copy of the nonnegative integers. Indeed, if $H$ is any elementary inverse semigroup, then $H^{1}$ is generated by a homomorphic image of the nonnegative integers, and so is a one-parameter inverse semigroup.

The main purpose of this paper is to describe all one-parameter inverse semigroups. In the process of doing this, we shall construct what we term the free oneparameter inverse semigroups $F_{P}$, one for each subgroup $G$ of $R$ and its associated semigroup $P$. The semigroup $F_{P}$ is the only inverse semigroup (up to isomorphism) generated by a subsemigroup isomorphic with $P$ which has the property that each homomorphism $f: P \rightarrow S$, an inverse semigroup, extends uniquely to a homomorphism $\bar{f}: F_{P} \rightarrow S$. In particular, every $H \in \mathscr{C}_{P}$ is a homomorphic image of $F_{P}$. We thus adopt the point of view that by describing $F_{P}$ and the lattice of congruences of $F_{P}$ for arbitrary $P$, we will have described all one-parameter inverse semigroups.

We shall assume a certain familiarity with the algebraic theory of semigroups, particularly inverse semigroups. (See Clifford and Preston [1].)

The existence and uniqueness of $F_{P}$ is a consequence of a theorem due to McAlister [3, Theorem 33]. We were greatly aided in the actual description of $F_{P}$

Presented to the Society, August 27, 1969; received by the editors June 10, 1969.

AMS 1970 subject classifications. Primary 20M10; Secondary 20M05, 22A15.

Key words and phrases. Inverse semigroup, one-parameter inverse semigroup, bicyclic semigroup, free semigroup, lattice of congruences, freely generated, bisimple inverse semigroup, Green's relations, normal congruence, group congruence, lattice of subgroups, kernel. 
by two results of Gluskin on elementary inverse semigroups [2, p. 24]. For the description of the congruences on $F_{P}$, the results of Reilly and Scheiblich in [4] proved useful.

Although this paper is primarily algebraic in nature, there is a natural topology on $F_{P}$ with respect to which $F_{P}$ is a topological inverse semigroup. This fact, together with several other comments of a topological nature, are included in remarks throughout the paper.

1. The free inverse semigroup on a set $X$. In this section we shall review some theory which has already been obtained by McAlister in [3].

If $S$ is an inverse semigroup generated by a subset $X$, then we say that $S$ is freely generated by $X$ provided each function from $X$ into an inverse semigroup extends to a homomorphism on $S$. One shows easily, using the fact that homomorphisms on inverse semigroups take inverses to inverses, that if $S$ is freely generated by $X$, then each function from $X$ into an inverse semigroup $T$ extends to a unique homomorphism from $S$ into $T$.

1.1. TheOREM. For any nonvoid $X$ there is one and only one inverse semigroup (up to isomorphism) $I_{X}$ freely generated by $X$.

Although it is not our intention to investigate them here, we remark that many interesting questions arise concerning the structure of $I_{X}$ and its lattice of congruences. For example, it is not difficult to show that the smallest group congruence on $I_{X}$ has the free group on $X$ as its quotient semigroup.

Now let $P$ be a fixed semigroup. Consider the class of pairs $(f, S)$ where $S$ is an inverse semigroup and $f$ is a homomorphism from $P$ into $S$ so that $f(P)$ generates $S$. Define two pairs $(f, S)$ and $(g, T)$ to be equivalent provided there is an isomorphism $\phi: S \stackrel{\text { onto }}{\longrightarrow} T$ so that $\phi f=g$. This is easily seen to be an equivalence relation on pairs. We call a pair $(f, S)$ a free pair provided given any pair $(g, T)$ there is a homomorphism $\phi: S \rightarrow T$ such that $\phi f=g$. It follows from the fact that two homomorphisms on an inverse semigroup which agree on a generating set are identical, that the homomorphism $\phi$ above is unique.

The next theorem establishes the existence and uniqueness of a free pair $(f, S)$.

1.2. THEOREM. There is an inverse semigroup $S$ and a homomorphism $f: P \rightarrow S$ such that $(f, S)$ is a free pair. Furthermore any two free pairs are equivalent. The homomorphism $f$ is 1-1 if and only if $P$ is embeddable in an inverse semigroup.

In case $f$ is $1-1$ we identify $P$ with $f(P)$ and call $S$ the inverse semigroup freely generated by the subsemigroup $P$ and denote $S$ by $F_{P}$. Note that $F_{P}$ is characterized by the property that any homomorphism from $P$ into an inverse semigroup extends to a unique homomorphism on $F_{P}$. In particular, any inverse semigroup generated by a homomorphic image of $P$ is isomorphic with a quotient semigroup of $F_{P}$. 
2. The free one-parameter inverse semigroups $F_{P}$. Let $G$ be a fixed subgroup of $R$ and let $P=\{x \in G \mid x \geqq 1\}, P_{0}=P \mid\{1\}$. In this section we shall describe fully the structure of the semigroups $F_{P}$ and $F_{P_{0}}$ freely generated by the subsemigroups $P$ and $P_{0}$ respectively.

First we construct a homomorphic image $B_{P}$ of $F_{P}$ which is a generalization of the bicyclic semigroup $B$. This construction is similar to the one found on p. 107 of Vol. 2 of [1]. Let $B_{P}=P \times P$ with the following operation:

$$
(x, y)(z, w)=(x z / y \wedge z, y w / y \wedge z)
$$

where $y \wedge z=\min \{y, z\}$. It is easily checked that the product of two elements of $B_{P}$ is an element of $B_{P}$. In fact we have the following consequence of Theorems 8.43 and 8.44 of Vol. 2 of [1]:

2.1. TheOREM. $B_{P}$ is a bisimple inverse semigroup which is generated by $P_{0} \times 1$.

2.2. THEOREM. The real number 1 is the identity for $F_{P}$. Furthermore $F_{P_{0}}$ does not have an identity and in fact is isomorphic with $F_{P} \mid\{1\}$. Thus $F_{P}$ is obtained from $F_{P_{0}}$ by adjoining an identity.

Proof. Since 1 is the identity of $P$ and $P$ generates $F_{P}, 1$ is the identity of $F_{P}$. Let $S$ denote the inverse subsemigroup of $F_{P}$ generated by $P_{0}$, and let $f$ be a homomorphism from $P_{0}$ into an inverse semigroup $T$. We assume $T$ has an identity $e$, for otherwise we could adjoin it. Then $f$ extends to a homomorphism $g: P \rightarrow T$ by defining $g(1)=e$. Now $g$ extends to a homomorphism $\bar{g}: F_{P} \rightarrow T$, and $\bar{g} \mid S$ is clearly the sought extension of $f$ to $S$. Thus $S$ is freely generated by $P_{0}$; that is, $S=F_{P_{0}}$. Now suppose $S$ has an identity $i$. Then there exist $x_{1}, x_{2}, \ldots, x_{n}$ in $P_{0}$ such that $i=x_{1}^{j_{1}} x_{2}^{j_{2}} \cdots x_{n}^{j_{n}}$ where $j_{k} \in\{1,-1\}$ for $k=1,2, \ldots, n$. Thus $x_{1}^{j_{1}} x_{1}^{-j_{1}}=x_{1}^{j_{1}} x_{1}^{-j_{1}} \cdot i$ $=i$ and hence, for some $x \in P_{0}, i=x x^{-1}$ or $i=x^{-1} x$. Suppose that $i=x x^{-1}$. Let $f: P_{0} \rightarrow P_{0} \times 1 \subseteq B_{P}$ be given by $f(t)=(t, 1)$. Then $f$ extends to a homomorphism $\bar{f}: S \rightarrow B_{P}$. Further $f(S)=B_{P}$ since $P_{0} \times 1$ generates $B_{P}$. Hence $\bar{f}(i)$ is an identity for $B_{P}$ and so $\bar{f}(i)=(1,1)$. But $\bar{f}(i)=\bar{f}\left(x x^{-1}\right)=\bar{f}(x) \bar{f}(x)^{-1}=(x, 1)(1, x)=(x, x)$ and $x \neq 1$. From this contradiction we conclude that $S=F_{P_{0}}$ does not have an identity. In particular $1 \notin S$. Suppose $x \in F_{P} \mid\{1\}$. Then there exist elements $x_{1}, x_{2}, \ldots, x_{n}$ of $P$ so that $x=x_{1}^{j_{1}} x_{2}^{j_{2}} \cdots x_{n}^{j_{n}}$ where $j_{k} \in\{1,-1\}$ for $k=1,2, \ldots, n$. In fact we may assume that $x_{k} \in P_{0}$ for $k=1,2, \ldots, n$ (this is true for at least one value of $k$ since $x \neq 1$ ). Thus $x \in S$ and we have shown that $S=F_{P} \mid\{1\}$. This completes the proof of this theorem.

An elementary inverse semigroup is defined to be an inverse semigroup generated by a single element. An elementary inverse semigroup may or may not be a oneparameter inverse semigroup depending on whether it has an identity; however we do have the following corollary.

2.3. Corollary. Suppose the given subgroup $G$ of $R$ is cyclic. Then $F_{P_{0}}$ is an elementary inverse semigroup with the property that every elementary inverse semigroup is a homomorphic image of $F_{P_{0}}$. 
Proof. This follows from 2.2 together with the fact that a homomorphism on the positive integers is determined by its value at 1 .

2.4. LEMMA. If $x \leqq y$ then

(i) $x y^{-1}=(y / x)^{-1} y y^{-1}$,

(ii) $y^{-1} x=y^{-1} y(y / x)^{-1}$,

(iii) $y x^{-1}=(y / x) x x^{-1}$,

(iv) $x^{-1} y=x^{-1} x(y / x)$.

Proof. To see (i), note that

$$
\begin{aligned}
x y^{-1} & =x((y / x) x)^{-1}=x x^{-1}(y / x)^{-1}=x x^{-1}(y / x)^{-1}(y / x)(y / x)^{-1} \\
& =(y / x)^{-1}(y / x) x x^{-1}(y / x)^{-1}=(y / x)^{-1} y y^{-1} .
\end{aligned}
$$

Part (ii) is proved similarly and (iii) and (iv) are trivial.

The next result is, in a sense, an analogue of a theorem of Gluskin [2, Lemma 1.2] and follows immediately from the above lemma.

2.5. Lemma. Let $x, y, z \in P$. Then the elements $x y^{-1} z$ and $x^{-1} y z^{-1}$ of $F_{P}$ can also be written as follows:

(i)

$$
\begin{aligned}
x y^{-1} z=x z / y & \text { if } y \leqq x, z, \\
=(y / x)^{-1} z & \text { if } x \leqq y \leqq z, \\
=x(y / z)^{-1} & \text { if } z \leqq y \leqq x, \\
=(y / x)^{-1} y(y / z)^{-1} & \text { if } x, z \leqq y . \\
x^{-1} y z^{-1}=(x z / y)^{-1} & \text { if } y \leqq x, z, \\
=x^{-1}(y / z) & \text { if } z \leqq y \leqq x, \\
=(y / x) z^{-1} & \text { if } x \leqq y \leqq z, \\
=(y / x) y^{-1}(y / z) & \text { if } x, z \leqq y .
\end{aligned}
$$

(ii)

(iii) There exist $a, b, c$ in $P$ such that $b \geqq a, c$ and $x^{-1} y z^{-1}=a b^{-1} c$.

Proof. Parts (i) and (ii) follow immediately from Lemma 2.4. Using (ii) we can write $x^{-1} y z^{-1}$ as $a b^{-1} c$ if we choose $a, b$ and $c$ as follows: if $y \leqq x, z$ let $a=1$, $b=x z / y, c=1$; if $z \leqq y \leqq x$, let $a=1, b=x, c=y / z$; if $x \leqq y \leqq z$, let $a=y / x, b=z, c=1$; and if $x, z \leqq y$, let $a=y / x, b=y, c=y / z$. In each case $b \geqq a, c$, and $a, b$ and $c$ are in $P$.

2.6. TheOREM. $F_{P}=P P^{-1} P=P^{-1} P P^{-1}$ and $F_{P_{0}}=P P_{0}^{-1} P=P^{-1} P_{0} P^{-1}$.

Proof. It is an immediate consequence of 2.5(i) that $P P^{-1} P \subset P^{-1} P P^{-1}$. Hence $P^{-1} P P^{-1}=\left(P P^{-1} P\right)^{-1} \subset\left(P^{-1} P P^{-1}\right)^{-1}=P P^{-1} P$ and so $P P^{-1} P=P^{-1} P P^{-1}$. Note also that $\left(P P^{-1} P\right)^{2}=\left(P P^{-1} P\right)\left(P P^{-1} P\right) \subset P\left(P^{-1} P P^{-1}\right) P=P\left(P P^{-1} P\right) P \subset P P^{-1} P$. Hence $P P^{-1} P$ is an inverse subsemigroup of $F_{P}$. Since $P \subset P P^{-1} P$, we obtain $F_{P}$ $=P P^{-1} P$. Now suppose $u \in F_{P_{0}}=F_{P} \mid\{1\}$. Then there exist $x, y, z \in P$ such that $u=x^{-1} y z^{-1}$. Now it follows from 2.5(iii) that there exist $a, b, c \in P$ with $b \geqq a, c$ so 
that $u=x^{-1} y z^{-1}=a b^{-1} c$. However, at least one of $a, b, c$ is not 1 , and so $b \neq 1$. This says that $u \in P P_{0}^{-1} P$. On the other hand, choose $x y^{-1} z$ in $P P_{0}^{-1} P$. Suppose $1=x y^{-1} z$. Note that $y \neq 1$. If $x=z=1$, then $y^{-1}=1$. So $y=1$ which is a contradiction. Thus, either $x \neq 1$ or $z \neq 1$. Without loss of generality, suppose $x \neq 1$. Now if $z=1$, then $1=x y^{-1} \in F_{P_{0}}$, which is a contradiction. So $z \neq 1$. Thus none of $x, y$, or $z$ is 1. Therefore $1=x y^{-1} z \in F_{P_{0}}$, another contradiction. Thus $x y^{-1} z \neq 1$; i.e., $x y^{-1} z \in F_{P_{0}}$. Hence $P P_{0}^{-1} P=F_{P_{0}}$.

2.7. THEOREM. Each element of $F_{P}$ can be written in one and only one way in the form $x y^{-1} z$ where $x, y, z \in P$ with $x, z \leqq y$. Refer to this as the canonical representation of elements of $F_{P}$. Then if $u, v \in F_{P}$ with canonical representations $u=x y^{-1} z$ and $v=r s^{-1}$, then $u v$ has as its canonical representation

$$
u v=(x z r / y \wedge z r)(y z r s /(y \wedge z r)(z r \wedge s))^{-1}(z r t / z r \wedge s) .
$$

Proof. Let $u \in F_{P}$. Then by 2.6 there are elements, $a, b, c \in P$ such that $u$ $=a^{-1} b c^{-1}$. Now using 2.5(iii) we can write $u=x y^{-1} z$ where $x, z \leqq y$. To show that the representation is unique, we make use of the semigroup $B_{P}$ defined earlier. Let $f, g: P \rightarrow B_{P}$ be the homomorphisms given by $f(x)=(x, 1)$ and $g(x)=(1, x)$. Let $\bar{f}$ and $\bar{g}$ be the extensions of $f$ and $g$ respectively to $F_{P}$. Now suppose that $u \in F_{P}$ has two representations $x y^{-1} z$ and $r s^{-1} t$ where $x, z \leqq y$ and $r, t \leqq s$. Then $\bar{f}\left(x y^{-1} z\right)$ $=f(x) f(y)^{-1} f(z)=(x, 1)(1, y)(z, 1)=(x, y / z)$ and similarly $\bar{f}\left(r s^{-1} t\right)=(r, s / t)$, $\bar{g}\left(x y^{-1} z\right)=(y / x, z)=\bar{g}\left(r s^{-1} t\right)=(s / r, t)$. Hence $r=x, s=y$ and $z=t$ and thus the representation is unique.

To establish the rule for multiplication, let $u, v \in F_{P}$ with representations (not necessarily canonical) $u=x y^{-1} z$ and $v=r s^{-1} t$. It then follows from 3.4(ii) that

$$
\begin{aligned}
u v & =x(y s / z r)^{-1} t & & \text { if } z r \leqq s, y, \\
& =x y^{-1}(z r t / s) & & \text { if } s \leqq z r \leqq y, \\
& =(x z r / y) s^{-1} t & & \text { if } y \leqq z r \leqq s, \\
& =(x z r / y)(z r)^{-1}(z r t / s) & & \text { if } s, y \leqq z r .
\end{aligned}
$$

Now since $y \wedge z r \leqq x z r$ and $z r \wedge s \leqq z r t$ it follows that $x z r /(y \wedge z r), z r t /(z r \wedge s)$, and $y z r t /((y \wedge z r)(z r \wedge s))$ are all in $P$. It is a simple matter to check using the four cases above that in fact,

$$
u v=(x z r / y \wedge z r)[y z r s /(y \wedge z r)(z r \wedge s)]^{-1}(z r t / z r \wedge s) .
$$

Further, if $x y^{-1} z$ and $r s^{-1} t$ are canonical; i.e. if $x, z \leqq y$ and $r, t \leqq s$ then it is easily checked that $x z r / y \wedge z r, z r t / z r \wedge s \leqq y z r s /(y \wedge z r)(z r \wedge s)$ and so the representation for the product above is canonical. This completes the proof.

2.8. CoROllaRY. The elements of $F_{P_{0}}=F_{P} \mid\{1\}$ consist precisely of those elements of $F_{P}$ whose canonical representation $x y^{-1} z$ is such that $y \neq 1$.

Proof. Let $u \in F_{P_{0}}$ and let $x y^{-1} z$ be its canonical representation. If $y=1$ then $x=z=1$ and so $u=1$. Hence $y \neq 1$. Conversely, if $x y^{-1} z \in F_{P}$ with $x, z \leqq y \neq 1$, then $x y^{-1} z \in P P_{0}^{-1} P=F_{P_{0}}$, by 2.6. Q.E.D. 
Using 2.7 and 2.8 we immediately obtain the following parametrization theorem for $F_{P}$ and $F_{P_{0}}$.

2.9. Corollary. Let $T_{P}=\{(x, y, z) \mid x, y, z \in P$ with $x, z \leqq y\}$. Define an operation on $T_{P}$ by

$$
(x, y, z)(r, s, t)=(x z r / y \wedge z r, y z r s /(y \wedge z r)(z r \wedge s), z r t / z r \wedge s) .
$$

Then the map $\phi: F_{P} \rightarrow T_{P}$ defined by $\phi(u)=(x, y, z)$ for $u \in F_{P}$ with canonical representation $u=x y^{-1} z$ is an isomorphism from $F_{P}$ onto $T_{P}$. Further if $T_{P_{0}}=T_{P} \mid\{(1,1,1)\}$, then $\phi \mid F_{P_{0}}$ is an isomorphism from $F_{P_{0}}$ onto $T_{P_{0}}$.

2.10. Remark. If $T_{P}$ is given the subspace topology from the product space $P \times P \times P$, where $P$ is given the subspace topology from $R$ with the usual topology, then it is easily seen that the multiplication and inversion on $T_{P}$ are continuous; that is, $T_{P}$ is a topological inverse semigroup. This follows from the fact that multiplication and inversion on $R$ and the $\wedge$ operation on $P$ are all continuous operations. Hence there is a natural topology on $F_{P}$ making $F_{P}$ into a topological inverse semigroup. Indeed, $F_{P}$ is freely generated by $P$ even in the topological sense; that is, any continuous homomorphism from $P$ into a topological inverse semigroup $S$ extends to a unique continuous homomorphism from $F_{P}$ into $S$.

The idempotent structure of $F_{P}$ is determined next.

2.11. Lemma. Let $u \in F_{P}$ with canonical representation $u=x y^{-1} z$. Then the canonical representation of $u^{-1}$ is $(y / z) y^{-1}(y / x)$.

Proof. Note $y / z, y / x \in P$. Also note $u^{-1}=z^{-1} y x^{-1}$. Hence by 2.5 (ii) $u^{-1}$ $=(y / z) y^{-1}(y / x)$.

For $x \in P$, let $e_{x}=x x^{-1}$ and $f_{x}=x^{-1} x$, and let $E=\left\{e_{x} \mid x \in P\right\}, F=\left\{f_{x} \mid x \in P\right\}$. Note $E, F \subseteq E_{P}$, the set of idempotents of $F_{P}$.

2.12. TheOREM. Let $u \in F_{P}$ with canonical representation $x y^{-1} z$. Then $u \in E_{P}$ if and only if $y=x z$. Furthermore, each element of $E$ can be written in one and only one way in the form $e_{x} f_{x}$ for some $x, z \in P$. Thus $E_{P}$ is the direct sum of the two subsemilattices $E$ and $F$. Also $e_{x} f_{y} \leqq e_{u} f_{v}$ if and only if $u \leqq x$ and $v \leqq y$.

Proof. Suppose $u \in E_{P}$ and $x y^{-1} z$ is the canonical representation of $u$. Then by 2.9, $u=u^{-1}=(y / z) y^{-1}(y / x)$. Hence $(y / z)=x$, that is, $y=x z$. On the other hand, if $y=z x$ then $x y^{-1} z=\left(x x^{-1}\right)\left(z^{-1} z\right)=e_{x} f_{z} \in E_{P}$. Hence to establish the last statement we need only show the uniqueness of the representation. So suppose $x, z, r, t \in P$ with $x x^{-1} z^{-1} z=e_{x} f_{z}=e_{r} f_{t}=r r^{-1} t^{-1} t$. Then, using the homomorphisms $\bar{f}$ and $\bar{g}$ of 2.7 we see that $f\left(x x^{-1} z^{-1} z\right)=f(x) f(x)^{-1} f(z)^{-1} f(z)=(x, 1)(1, x)(1, z)(z, 1)=(x, x)$ $=\bar{f}\left(r r^{-1} t t^{-1}\right)=(r, r)$ and similarly $\bar{g}\left(x x^{-1} z^{-1} z\right)=(z, z)=\bar{g}\left(r r^{-1} t^{-1} t\right)=(t, t)$. Hence $x=r$ and $z=t$. The last assertion follows easily upon noting that $e_{x} e_{u}=e_{x \vee u} \cdot 2.13$ follows immediately from 2.12 and the fact that $F_{P_{0}}=F_{P} \mid\{1\}$. 
2.13. Corollary. The idempotents of $F_{P_{0}}$ are precisely those elements of $F_{P}$ which can be written (uniquely) in the form $e_{x} f_{z}$ where $\{x, z\} \cap P_{0} \neq \varnothing$.

Next we determine Green's relations (confer with [1]) on $F_{P}$.

2.14. TheOREM. Let $u, v \in F_{P}$ with canonical representations $u=x y^{-1} z$ and $v=r s^{-1} t$. Then

(i) $u \mathscr{R} v$ if and only if $x=r$ and $y=s$,

(ii) $u \mathscr{L} v$ if and only if $y=s$ and $z=t$,

(iii) $u \mathscr{H} v$ if and only if $x=r, y=s$ and $z=t$,

(iv) $u \mathscr{D} v$ if and only if $y=s$.

Proof. (i) We know $u \mathscr{R} v$ if and only if $u u^{-1}=v v^{-1}$. But

$$
u u^{-1}=\left(x y^{-1} z\right)\left((y / z) y^{-1}(y / x)\right)=x y^{-1}(y / x)
$$

and similarly $v v^{-1}=r s^{-1}(s / t)$. Hence by $2.7 u u^{-1}=v v^{-1}$ if and only if $x=r$ and $y=s$.

(ii) Analogous to (i).

(iii) Follows immediately from (i) and (ii).

(iv) Suppose $u \mathscr{D} v$. Then there is an element $w$ of $F$ with $u \mathscr{R} w$ and $w \mathscr{L} v$. Let $a b^{-1} c$ be the canonical representation of $w$. Then by (i) $y=b$ and by (ii) $b=s$. Hence $y=s$. On the other hand, if $y=s$ let $w=x y^{-1} t$. Then $u \mathscr{R} w$ by (i) and $w \mathscr{L} v$ by (ii). Hence $u \mathscr{D} v$. This completes the proof of 2.12 .

From 2.14 we get that there is a $\mathscr{D}$-class $D_{y}$ for each element $y$ of $D: D_{y}$ $=\left\{x y^{-1} z \mid x, z \in P\right.$ with $\left.x, z \leqq y\right\}$. Note also that $E_{P} \cap D_{y}=\left\{e_{x} f_{z} \mid x z=y\right\}$. Hence the $\mathscr{D}$-class $D_{y}$ can be pictured as in Figure 1.

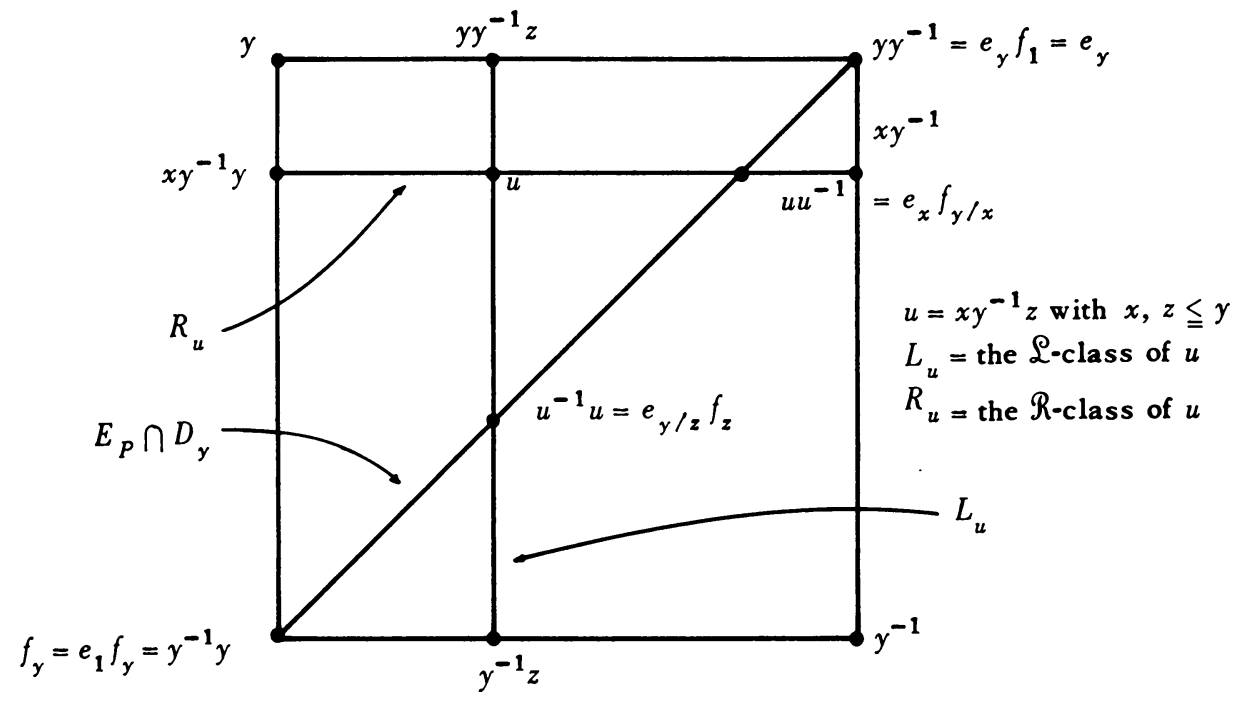

FIGURE 1 
It may be helpful to visualize $F_{P}$ as in Figure 2 .

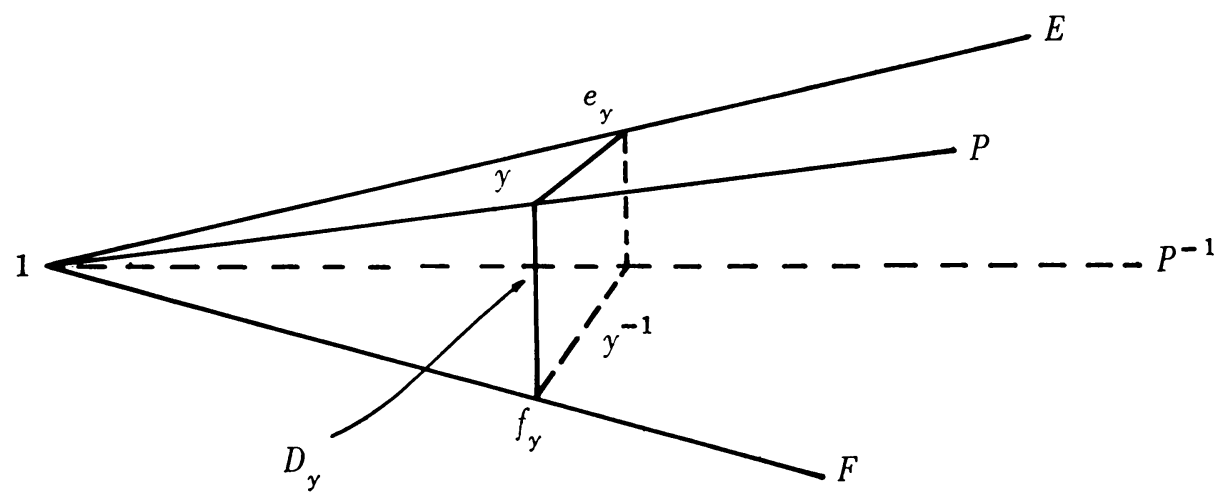

Figure 2

Note that the idempotents of $F_{P}$ lie in a plane which cuts $F_{P}$ into two pieces.

Next we determine the ideal structure of $F_{P}$. For $y \in R$, let

$$
I_{y}=\bigcup\left\{D_{t} \mid t \geqq y \text { and } t \in P\right\}
$$

and let

$$
I_{y}^{\circ}=\bigcup\left\{D_{t} \mid t>y \text { and } t \in P\right\} .
$$

2.15. TheOREM. For each $y \in P, I_{y}$ and $I_{y}^{\circ}$ are ideals of $F_{P}$. Conversely, if $I$ is an ideal of $F_{P}$, then there is an element $y \geqq 1$ of $R$ such that $I=I_{y}$ or $I=I_{y}^{\circ}$. Consequently the ideals of $F_{P}$ are totally ordered with respect to set inclusion.

Proof. The fact that $I_{y}$ and $I_{y}^{\circ}$ are ideals of $F_{P}$ follows readily from the rule for multiplication expressed in 2.7. On the other hand, if $I$ is an ideal of $F_{P}$, then let $y$ denote the greatest lower bound of the set of all $t \in P$ such that $D_{t} \cap I \neq \varnothing$. It is not difficult to show that if $D_{t} \cap I \neq \varnothing$, then $D_{t t_{1}} \subset I$ for all $t_{1} \in P$, and hence $I=I_{y}$ if $D_{y} \cap I \neq \varnothing$ or $I=I_{y}^{\circ}$ if $D_{y} \cap I=\varnothing$. Q.E.D.

2.16. REMARK. If we give $F_{P}$ the natural topology described in 2.10 then the closed ideals are the ones which can be written in the form $I_{y}$.

3. The lattice of congruences on $F_{P}$. In this section as in the last, $G$ is an arbitrary subgroup of $R$, the multiplicative group of positive reals, and $P=\{x \in G \mid x \geqq 1\}$. We shall describe here the structure of the lattice of congruences on the free one-parameter inverse semigroup $F_{P}$, and hence obtain a description of every one-parameter inverse semigroup.

The set $\Lambda(S)$ of congruences on a semigroup $S$ is well known to be a complete lattice with respect to the operations

$$
\sigma \wedge \rho=\sigma \cap \rho \text { and } \sigma \vee \rho=\bigcap\{\delta \in \Lambda(S) \mid \cup \rho \sigma \subset \delta\} .
$$

The largest (resp. smallest) congruence on $S$, which is $S^{2}=S \times S$ (resp. $\Delta S^{2}$ $=\{(x, x) \mid x \in S\}$ ), is denoted by 1 (resp. 0 ). The $\theta$ relation on $\Lambda(S)$, first defined and studied on regular semigroups $S$ by Reilly and Scheiblich [4] provides a useful aid in visualizing $\Lambda(S)$. The relation is defined by $\sigma \theta \rho$ if and only if $\sigma \cap E^{2}=$ 
$\rho \cap E^{2}$, where $E$ is the set of idempotents on $S$. It is shown in [4] that if $S$ is an inverse semigroup, then $\theta$ is a lattice congruence on $\Lambda(S)$. The $\theta$-class of 1 is the set of group congruences on $S$; the $\theta$-class of 0 is the set of idempotent-separating congruences; in general, each $\theta$-class is a complete lattice of commuting congruences on $S$.

A congruence $\omega$ on $E$, the idempotents of an inverse semigroup $S$, is normal provided whenever $e \omega f$, then $x e x^{-1} \omega x f x^{-1}$ for all $x \in S$. The normal congruences on $E$ are precisely those congruences $\omega$ on $E$ such that $\omega=\sigma \cap E^{2}$ for some $\sigma \in \Lambda(S)$. In fact one sees that $\Lambda(S) / \theta$ is isomorphic with the lattice of normal congruences on $E$, under the map induced by the map from $\Lambda(S)$ to the normal congruences on $E$ given by $\sigma \rightarrow \sigma \cap E^{2}$.

As a first step in describing $\Lambda\left(F_{P}\right)$, we shall determine the normal congruences on $E_{P}$, the set of idempotents of $F_{P}$. Recall 2.12, which says that $E_{P}$ is the direct sum of $E=\left\{x x^{-1} \mid x \in P\right\}$ and $F=\left\{x^{-1} x \mid x \in P\right\}$.

3.1. Lemma. Let $x, y, t \in P$. Then

$$
\begin{aligned}
& t e_{x} f_{y} t^{-1}=\left\{\begin{array}{ll}
e_{t x} f_{y / t} & \text { if } t \leqq y \\
e_{l x} & \text { if } y \leqq t
\end{array}\right\}=e_{x t} f_{y / y \wedge t}, \\
& t^{-1} e_{x} f_{y} t=\left\{\begin{array}{ll}
e_{x i l} f_{t y} & \text { if } t \leqq x \\
f_{t y} & \text { if } x \leqq t
\end{array}\right\}=e_{x / x \wedge t} f_{t y} .
\end{aligned}
$$

Proof. This follows from the rule for multiplication expressed in 2.7.

Let $A$ and $B$ denote the relations on $E_{P}$ defined by $e_{x} f_{y} A e_{r} f_{s}$ if and only if $x=r$ and $e_{x} f_{y} B e_{r} f_{s}$ if and only if $y=s$. These are clearly congruence relations on $E_{P}$. Furthermore, it is also clear that $A \vee B=E_{P}^{2}$ and $A \wedge B=\Delta E_{P}^{2}$. Let $I$ be an ideal of $F_{P}$, and let $I A=\left(A \cap I^{2}\right) \cup \Delta E_{P}^{2}, I B=\left(B \cap I^{2}\right) \cup \Delta E_{P}^{2}$, and $I E_{P}^{2}=\left(E_{P}^{2} \cap I^{2}\right)$ $\cup \Delta E_{P}^{2}$. We see immediately that $I A, I B$, and $I E_{P}^{2}$ are all congruences on $E_{P}$ also.

3.2. THEOREM. Each of the above congruences on $E_{P}$ is normal. As a set of normal congruences, they form a lattice with the structure as indicated in the diagram below:

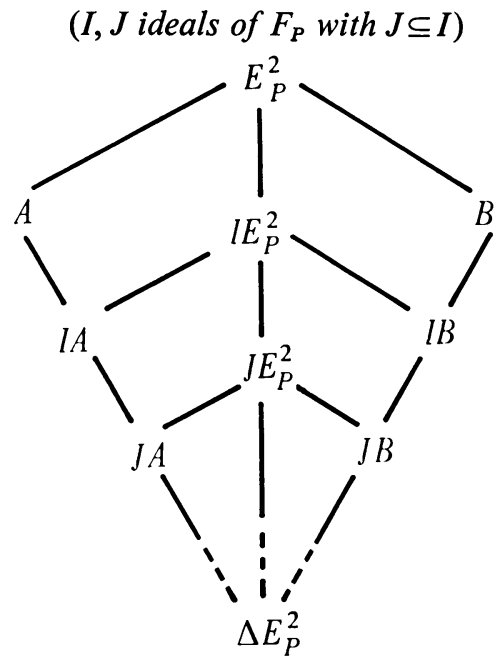

FIGURE 3 
Proof. If $I$ is an ideal of $F_{P}$ and $\omega$ is a normal congruence on $E_{P}$, then $I \omega=\left(\omega \cap I^{2}\right) \cup \Delta E_{P}^{2}$ is clearly a normal congruence on $E_{P}$, since it is the intersection of the two normal congruences $\omega$ and $\left(I^{2} \cap E_{P}^{2}\right) \cup \Delta E_{P}^{2}$. Hence the only assertion requiring proof is that $A$ and $B$ are normal. To see this, let $u=a b^{-1} c \in F_{P}$ and note that by 3.1

$$
u e_{x} f_{y} u^{-1}=e_{a c x / b \wedge c x} f_{(b y / y \wedge c) i(a \wedge[b y /(y \wedge c)])} .
$$

From this we see that $A$ and $B$ are normal. Q.E.D.

3.3. Lemma. Suppose $\omega$ is a normal congruence on $E_{P}$, and suppose $x_{0}, y_{0}, t_{0} \in P$ with $t_{0} \neq 1$. Let I denote the ideal $I_{x_{0} y_{0}}=\bigcup\left\{D_{t} \mid t \geqq x_{0} y_{0}\right\}$ of $F_{P}$. Then

(i) if $e_{x_{0}} f_{y_{0}} \omega e_{x_{0}} f_{y_{0} t_{0}}$, then $I A \subseteq \omega$,

(ii) if $e_{x_{0}} f_{y_{0}} \omega e_{x_{0} t_{0}} f_{y_{0}}$, then $I B \subseteq \omega$.

Proof. (i) Suppose $x, y, t \in P$ with $x y \geqq x_{0} y_{0}$. We wish to show that $e_{x} f_{y} \omega e_{x} f_{y t}$. Note that $e_{x} f_{y}=x f_{x y} x^{-1}$ and $e_{x} f_{y t}=x f_{x y t} x^{-1}$; hence the result follows if $f_{x y} \omega f_{x y t}$. To see this, first note that $f_{x_{0} y_{0}}=x_{0}^{-1} e_{x_{0}} f_{y_{0} x_{0}} \omega x_{0}^{-1} e_{x_{0}} f_{y_{0} t_{0}} x_{0}=f_{x_{0} y_{0} t_{0}}$. Hence $f_{x_{0} y_{0} t_{0}}=t_{0}^{-1} f_{x_{0} y_{0}} t_{0} \omega t_{0}^{-1} f_{x_{0} y_{0} t_{0}} t_{0}=f_{x_{0} y_{0} t_{0}}$, and so $f_{x_{0} y_{0}} \omega f_{x_{0} y_{0} t_{0}^{2}}$. Inductively, we have that $f_{x_{0} y_{0}} \omega f_{x_{0} y_{0} t_{0}^{n}}$ for each positive integer $n$. Now choose $n$ so large that $x_{0} y_{0} t_{0}^{n}$ $\geqq x y t \geqq x y$. Then since $\omega$ is a congruence on $E_{P}$,

$$
f_{x y}=f_{x y} \cdot f_{x_{0} y_{0}} \omega f_{x y} \cdot f_{x_{0} y_{0} t_{0}^{n}}=f_{x_{0} y_{0} t_{0}^{n}}
$$

and

$$
f_{x y t}=f_{x y t} \cdot f_{x_{0} y_{0}} \omega f_{x y t} \cdot f_{x_{0} y_{0} t_{0}^{n}}=f_{x_{0} y_{0} t_{0}^{n}} .
$$

Hence $f_{x y} \omega f_{x y t}$ and the proof of (i) is complete. The proof of (ii) is analogous.

3.4. THEOREM. Let $\omega$ be a nonzero normal congruence on $E_{p}$. Then there is an ideal I of $F_{P}$ such that $\omega$ is one of the congruences $I A, I B$, or IE $E_{P}^{2}$. Consequently the lattice shown in 3.2 is the lattice of all normal congruences on $E_{P}$.

Proof. Since $\omega \neq \Delta E_{P}^{2}$, there exist $x, y, r, s \in P$ with $x \neq r$ or $y \neq s$ such that $e_{x} f_{y} \omega e_{r} f_{s}$. Suppose $x \neq r$; say $x<r$. Then since $e_{x} f_{y v s}=e_{x} f_{y}\left(f_{y v s}\right) \omega e_{r} f_{s}\left(f_{y v s}\right)$ $=e_{r} f_{y \vee s}$, we have by 3.3 that $I_{x(s \vee y)} B \subseteq \omega$. Similarly, if $y<s$, then $I_{(x \vee r) y} A \subseteq \omega$. In any event, at least one of the sets $L=\left\{t \in P: I_{t} A \subseteq \omega\right\}$ and $R=\left\{T \in P: I_{t} B \subseteq \omega\right\}$ is nonvoid.

Suppose $R=\varnothing$ and $L \neq \varnothing$. Let $I_{L}=\bigcup\left\{I_{t}: t \in L\right\}$ and note that $I_{L} A$ $=\bigcup\left\{I_{l} A: t \in L\right\} \subseteq \omega$. So let $e_{x} f_{y} \omega e_{r} f_{s} ; x=r$, otherwise $R \neq \varnothing$. Assume $y<s$. Then $\left(e_{x} f_{y}, e_{r} f_{s}\right) \in I_{x y} A$. But by $3.3, I_{x y} A \subseteq \omega$ so $x y \in L$; hence $I_{x y} A \subseteq I_{L} A$. Therefore $\omega=I_{L} A$. By an analogous argument we conclude that if $L=\varnothing$, then $R \neq \varnothing$, so $I_{R} B$ $=\omega$ where $I_{R}=\bigcup\left\{I_{t}: t \in R\right\}$.

If neither $L$ nor $R$ is void, then we claim $L=R$ and $\omega=I_{L} E_{P}^{2}$. To see that $L=R$, let $t \in L$. Choose any $t_{0} \in R$. Then $\left(e_{t} f_{1}, e_{t} f_{t_{0}}\right) \in I_{t} A \subseteq \omega$ as $t \in L$; also $\left(e_{t} f_{t_{0}}, e_{t t_{0}} f_{t_{0}}\right)$ $\in I_{t_{0}} B \subseteq \omega$ and $\left(e_{t t_{0}} f_{t_{0}}, e_{t t_{0}} f_{1}\right) \in I_{t} A \subseteq \omega$. So $\left(e_{t} f_{1}, e_{t t_{0}} f_{1}\right) \in \omega$. By 3.3 we conclude that $I_{t} B \subseteq \omega$; i.e. $t \in R$. Thus $L \subseteq R$. Similarly $R \subseteq L$. So $L=R$. 
Note that $I_{L} E_{P}^{2} \subseteq \omega$ since $I_{L} A \subseteq \omega$ and $I_{R} B \subseteq \omega$, and $I_{L} A \vee I_{L} B=I_{L} E_{P}^{2}$. Now suppose $e_{x} f_{y} \omega e_{r} f_{s}$. If $x=r$ and $y=s$, then $\left(e_{x} f_{y}, e_{r} f_{s}\right) \in \Delta E_{P}^{2} \subseteq I_{L} E_{P}^{2}$. Without loss of generality assume $x \neq r$, say $x>r$. If $y=s$, then $\left(e_{x} f_{y}, e_{r} f_{y}\right) \in \omega$, so $I_{r y} B \subseteq \omega$. Thus $I_{r y} B \subseteq I_{L} E_{P}^{2}$, so $\left(e_{x} f_{y}, e_{r} f_{s}\right)=\left(e_{x} f_{y}, e_{r} f_{y}\right) \in I_{L} E_{P}^{2}$. Similarly for the case $x<r$. A similar argument shows if $x=r$ and $y \neq s$, then $\left(e_{x} f_{y}, e_{r} f_{s}\right) \in I_{L} E_{P}^{2}$. Now if $x \neq r$ and $y \neq s$, w.l.o.g. assume $x>r$. Then $e_{x} f_{y} \omega e_{x} f_{s}$, and hence $e_{x} f_{s} \omega e_{r} f_{s}$. By 3.3, this implies $I_{x(y \wedge s)} A \subseteq \omega$ and $I_{r s} B \subseteq \omega$, so $I_{x}(y \wedge s) A$ and $I_{r s} B \subseteq I_{L} E_{P}^{2}$. Therefore, $e_{x} f_{y}\left(I_{L} E_{P}^{2}\right) e_{x} f_{s}\left(I_{L} E_{P}^{2}\right) e_{r} f_{s}$, so $\left(e_{x} f_{y}, e_{r} f_{s}\right) \in I_{L} E_{P}^{2}$, and $\omega \subseteq I_{L} E_{P}^{2}$. This completes the proof.

Now that we have determined the lattice of normal congruences on $E_{P}$ (and hence the lattice $\Lambda\left(F_{P}\right) / \theta$, we concentrate on determining each $\theta$-class of $\Lambda\left(F_{P}\right)$. If $\omega$ is a normal congruence on $E_{P}$ then the $\theta$-class belonging to $\omega$ is the set of all congruences $\sigma \in \Lambda\left(F_{P}\right)$ such that $\sigma \cap E_{P}^{2}=\omega$.

Let $I$ be an arbitrary ideal of $F_{P}$. In the next three theorems we shall determine the $\theta$-class belonging to $I E_{P}^{2}$. Let $f$ denote the inclusion map of $P$ into $G$ and let $\bar{f}$ denote the extension of $f$ to $F_{P}$. Note that $\bar{f}\left(x y^{-1} z\right)=x z / y$, and that $\bar{f} \mid I$ is onto $G$.

3.5. THEOREM. A congruence $\sigma$ on $I$ is a group congruence if and only if there is a subgroup $N$ of $G$ such that for each $u, v \in I$ (with canonical representations $u=x y^{-1} z$, $\left.v=r s^{-1} t\right), u \sigma v$ if and only if $x z s / r t y \in N$.

Proof. Let $\sigma$ be a group congruence on $I$, and consider the following diagram:

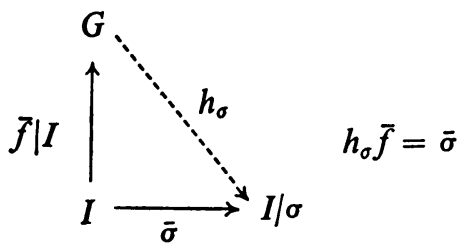

In order to check that the homomorphism $h_{\sigma}$ exists, we note that if $\bar{f} \mid I\left(x y^{-1} z\right)$ $=\bar{f} \mid I\left(r s^{-1} t\right)$, then $x z s=r t y$. Hence $\bar{\sigma}\left(x y^{-1} z\right)=\bar{\sigma}\left(r s^{-1} t\right)$. Since $\bar{f} \mid I$ is onto, there is a unique homomorphism induced which we call $h_{\sigma}$. Now let $N=\operatorname{ker} h_{\sigma}$ and note that $x y^{-1} z \sigma r s^{-1} t$ if and only if $\bar{\sigma}\left(x y^{-1} z\right)=\bar{\sigma}\left(r s^{-1} t\right)$ if and only if $h_{\sigma} \bar{f}\left(x y^{-1} z\right)=h_{\sigma} \bar{f}\left(r s^{-1} t\right)$ if and only if $h_{\sigma}(x z / y)=h_{\sigma}(r t / s)$ if and only if $x z / y \div r t / s=x z s / r t y \in \operatorname{ker} h_{\sigma}=N$.

Conversely suppose $N$ is a subgroup of $G$. Let $\sigma_{N}$ be the relation on $I$ defined by $x y^{-1} z \sigma_{N} r s^{-1} t$ if and only if $x z s / r t y \in N$, where $y \geqq x, z$ and $s \geqq r, t$ and $x y^{-1} z$, $r s^{-1} t \in I$. It is readily checked that $\sigma_{N}$ is a congruence on $I$ using the fact that $N$ is a group.

To see that $\sigma_{N}$ is a group congruence we need only show $I / \sigma_{N}$ has only one idempotent. So let $e, f$ be idempotents in $I$. Then by $2.10, e=x(x z)^{-1} z$ and $f=r(r t)^{-1} t$ for some $x, z, r$, and $t$ in $P$. Since $x z(r t) / r t(x z)=1 \in N$ we have that $e \sigma_{N} f$. Thus $I / \sigma_{N}$ is a group. 
3.6. TheOREM. The correspondences $\sigma \rightarrow \operatorname{ker} h_{\sigma}$ and $N \rightarrow \sigma_{N}$ described in 3.1 between the lattice of group congruences on I and the lattice of subgroups of $G$ are mutually inversive lattice isomorphisms.

Proof. Let $\sigma$ be a group congruence on $I$, and let $\delta=\sigma_{\text {ker } h_{\sigma}}$. Now as in 3.5 $x y^{-1} z \sigma r s^{-1} t$ if and only if $x z s / r t y \in \operatorname{ker} h_{\sigma}$. But from the definition of $\delta$, $x y^{-1} z \delta r s^{-1} t$ if and only if $x z s / r t y \in \operatorname{ker} h_{\sigma}$. Hence $\sigma_{\text {ker } h_{\sigma}}=\sigma$. On the other hand, let $N$ be a subgroup of $G$. Let $u, v \in I$ with canonical representations $u=x y^{-1} z$ and $v=r s^{-1} t$. Now $u \sigma_{N} v$ if and only if $x z s / r t y \in N$. Also using the induced homomorphism $h_{\sigma_{N}}, u \sigma_{N} v$ if and only if $x z s / r t y \in \operatorname{ker} h_{\sigma_{N}}$. Hence $N=\operatorname{ker} h_{\sigma_{N}}$. Hence the correspondences are mutually inversive functions. To complete the proof we need only show that the correspondence $N \rightarrow \sigma_{N}$ is a lattice homomorphism.

Let $N$ and $M$ be subgroups of $G$. It will suffice to show that $N \subseteq M$ if and only if $\sigma_{N} \subseteq \sigma_{M}$. Now it is clear that $N \subseteq M$ implies $\sigma_{N} \subseteq \sigma_{M}$. Conversely if $\sigma_{N} \subseteq \sigma_{M}$ let $x$ be in $N$ with $x=y / z$ such that $y, z \in P$. Then $(1, y, 1) \sigma_{N}(1, z, 1)$ implies $(1, y, 1) \sigma_{M}(1, z, 1)$. Thus $x \in M$ and $N \subseteq M$. This completes the proof of 3.6.

3.7. THEOREM. The $\theta$-class belonging to the normal congruence $I E_{P}^{2}$ is isomorphic with the lattice of subgroups of $G$ under the correspondence $N \rightarrow \sigma_{N} \cup \Delta F_{P}^{2}$.

Proof. Let $\Gamma$ denote the $\theta$-class belonging to $I E_{P}^{2}, \Omega$ the lattice of subgroups of $G$, and $\Delta$ the lattice of group congruences on $I$. By 3.6 the function from $\Omega$ onto $\Delta$ taking $N$ to $\sigma_{N}$ is a lattice isomorphism. Hence we only need show that the function from $\Delta$ to $\Gamma$ taking $\delta$ to $\delta \cup \Delta F_{P}^{2}$ is a 1-1 onto lattice isomorphism.

To see that this function is $1-1$ and onto, let $\delta \cup \Delta F_{P}^{2}=\delta^{\prime}$ for $\delta \in \Delta$ and $\rho \cap I^{2}$ $=\rho^{*}$ for $\rho \in \Gamma$. Clearly $\delta^{\prime} \in \Gamma$ and $\rho^{*} \in \Delta$. Also one sees without difficulty that $\left(\delta^{\prime}\right)^{*}=\delta$, for $\delta \in \Delta$. On the other hand if $\rho \in \Gamma$, then to show that $\left(\rho^{*}\right)^{\prime}=\rho$ we need only show that whenever $u, v \in F_{P}$ with $u \neq v$ and $u \rho v$ then $u, v \in I$. We consider two cases: (1) If $u \notin I, v \in I$, then $u u^{-1} \notin I$ and $v v^{-1} \in I$. Also $u u^{-1} \rho v v^{-1}$. However this is impossible since $\rho \cap E_{P}^{2}=I E_{P}^{2}$. (2) If $u \notin I, v \notin I$, then $u u^{-1}, v v^{-1}, u^{-1} u$, $v^{-1} v \notin I$; but $u u^{-1} \rho v v^{-1}$, so $u u^{-1}=v v^{-1}$ since $\rho \cap E_{P}^{2}=I E_{P}^{2}$. Similarly $u^{-1} u$ $=v^{-1} v$. However this implies that $u$ and $v$ are $\mathscr{H}$ related and so by 2.14 we conclude that $u=v$, a contradiction. This shows that $\left(\rho^{*}\right)^{\prime}=\rho$. Hence the functions $\delta \rightarrow \delta^{\prime}$ and $\rho \rightarrow \rho^{*}$ are mutually inversive functions; and thus $\sigma_{N} \rightarrow \sigma_{N} \cup \Delta F_{P}^{2}$ is a 1-1 onto function.

To see that it is a lattice isomorphism, let $\delta, \sigma \in \Delta$. Then $\delta \vee \sigma=\delta \circ \sigma$, since $\delta \circ \sigma=\sigma \circ \delta$. Also $\delta^{\prime} \vee \sigma^{\prime}=\delta^{\prime} \circ \sigma^{\prime}$ according to [4]. So $(\delta \vee \sigma)^{\prime}=(\delta \circ \sigma) \cup \Delta F_{P}^{2}$, and $\delta^{\prime} \vee \sigma^{\prime}=\left(\delta \cup \Delta F_{P}^{2}\right) \circ\left(\sigma \cup \Delta F_{P}^{2}\right)$. From this it follows that $(\delta \vee \sigma)^{\prime}=\delta^{\prime} \vee \sigma^{\prime}$; hence $\sigma_{N} \rightarrow \sigma_{N} \cup \Delta F_{P}^{2}$ preserves $\vee$. Since the inverse of this function clearly preserves $\wedge$, we conclude that $\sigma_{N} \rightarrow \sigma_{N} \cup \Delta F_{P}^{2}$ is a lattice isomorphism.

3.8. Corollary. For each subgroup $N$ of $G$, let $\sigma^{N}$ denote the relation on $F_{P}$ defined by $u \sigma^{N} v$ if and only if $u=v$, or $u, v \in I$ and $x z s / r t y \in N$, where $x y^{-1} z$ and $r s^{-1} t$ are the canonical representations of $u$ and $v$ respectively. Then $\sigma^{N}$ is a member 
of the $\theta$-class belonging to $I E_{P}^{2}$. Furthermore if $M$ is a subgroup of $G$ then $\sigma^{N} \vee \sigma^{M}$ $=\sigma^{N M}$ and $\sigma^{N} \cap \sigma^{M}=\sigma^{N \cap M}$.

Now we shall determine the $\theta$-class belonging to $I A$ and $I B$. It turns out that they are both degenerate. Let $g, h: P \rightarrow B_{P}$ be the homomorphisms given by $g(x)$ $=(x, 1)$ and $h(x)=(1, x)$. Let $\bar{g}, \bar{h}: F_{P} \rightarrow B_{P}$ denote the extensions of $g$ and $h$, and let $\alpha, \beta$ be the congruences on $F_{P}$ determined by $\bar{g}, \bar{h}$ respectively. Note that $u \alpha v(u \beta v)$ if and only if $x=r$ and $y t=s z(z=t$ and $y r=s x)$ where $x y^{-1} z$ and $r s^{-1} t$ are the canonical representations of $u$ and $v$. Let $I \alpha=\left(\alpha \cap I^{2}\right) \cup \Delta F_{P}^{2}$ $\left(I \beta=\left(\beta \cap I^{2}\right) \cup \Delta F_{P}^{2}\right)$. It is readily checked that $I \alpha(I \beta)$ is a congruence on $F_{P}$ lying in the $\theta$-class belonging to $I A(I B)$.

3.9. THEOREM. The $\theta$-class belonging to IA $(I B)$ has $I \alpha(I \beta)$ as its only member.

Proof. Let $\Gamma$ denote the $\theta$-class belonging to $I A$, and let $\rho$ and $\sigma$ denote the largest and smallest elements of $\Gamma$ respectively. It follows from Theorem 4.2 of [4] that for $u, v \in F_{P}$ with canonical representations $x y^{-1} z$ and $r s^{-1} t$ respectively that $u \sigma v$ if and only if $u u^{-1}(I A) v v^{-1}$ and $e u=e v$ for some $e \in E_{P}$ such that $e I A u u^{-1}$. To prove the theorem we need only show that $u \rho v$ implies $u \sigma v$. So suppose $u \rho v$. Then $u^{-1} \rho v^{-1}$ so $u u^{-1} \rho v v^{-1}$. Thus $e_{x} f_{y / x}=u u^{-1}(I A) v v^{-1}=e_{r} f_{s / r}$ and so $x=r$. Also $e_{y / z} f_{z}=u^{-1} u(I A) v^{-1} v=e_{s / t} f_{t}$ and so $y t=s z$. Now let $e=e_{x} f_{s y}$ and note that $e u=e v$ and $e I A u u^{-1}$. Hence $u \sigma v$, and we conclude that $\sigma=\rho=I \alpha$. The proof that the $\theta$-class belonging to $I B$ contains only $I \beta$ is analogous.

The following corollary sums up the information contained in 3.7 and 3.9. For an arbitrary ideal $I$ of $F_{P}$ and an arbitrary congruence $\sigma$ on $F_{P}$, let $I \sigma$ denote the congruence $\left(\sigma \cap I^{2}\right) \cup \Delta F_{P}^{2}$ on $F_{P}$. The top of $\Lambda\left(F_{P}\right), T$, is the set of group congruences on $F_{P}$ together with the two congruences $\alpha$ and $\beta$.

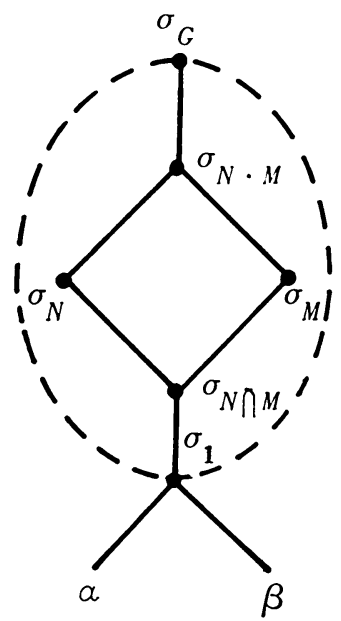

Figure 4 
3.10. COROLlaRY. Every nonzero congruence $\sigma$ on $F_{P}$ can be written uniquely in the form $I \delta$ for some $\delta \in T$ and some ideal $I$ of $F_{P}$. Furthermore for ideals $I$ and $J$ of $F_{P}$ and $\gamma$ and $\delta$ in $T, I \gamma \subset J \delta$ if and only if $I \subset J$ and $\gamma \subset \delta$.

3.11. Remark. If we consider $F_{P}$ with the topology described in 2.10 , then it is natural to ask what the closed congruences on $F_{P}$ are. It is not hard to see that 1,0 , $\alpha$ and $\beta$ are closed. Also the group congruence $\sigma_{N}$ is closed if and only if $N$ is cyclic, and if $I$ is an ideal of $F_{P}$ and $\sigma \in T$ then $I \sigma$ is closed if and only if $I$ is closed and $\sigma$ is closed.

Several additional pieces of information can be obtained from the preceding theorems. We state them below.

3.12. Corollary. $\Lambda\left(F_{P}\right)$ is a nonmodular lattice.

3.13. Corollary. All one-parameter inverse semigroups except those of the form $F_{P}$ have a kernel (i.e. minimal ideal). In particular, if $I$ is an ideal of $F_{P}$ then $F_{P} / I \alpha$ and $F_{P} / I \beta$ have a kernel isomorphic with $B_{P}$ and $F_{P} / I \sigma_{N}$ has a kernel isomorphic with $G / N$.

3.14. CoRollary. The lattice of congruences on $F_{P_{0}}$ is isomorphic with the complement of the top of $\Lambda\left(F_{P}\right)$ under the mapping $\sigma \rightarrow \sigma \cup\{(1,1)\}$.

\section{REFERENCES}

1. A. H. Clifford and G. B. Preston, The algebraic theory of semigroups. Vols. 1, 2, Math. Surveys, no. 7, Amer. Math. Soc., Providence, R. I., 1961, 1967. MR 24 \#A2627; MR 36 \#1558.

2. L. M. Gluskin, Elementary generalized groups, Mat. Sb. 41 (83) (1957), 23-36. (Russian) MR 19, 836.

3. D. B. McAlister, A homomorphism theorem for semigroups, J. London Math. Soc. 43 (1968), 355-366. MR 37 \#329.

4. N. R. Reilly and H. E. Scheiblich, Congruences on regular semigroups, Pacific J. Math. 23 (1967), 349-360. MR 36 \#2725.

UNIVERSity OF KeNTUCKy, LeXiNGTon, KentUCKy 40506 